\title{
Pierre Bézier et les machines transfert Renault (1940- 1960). Quand le cinéma ne suffit pas à consacrer un héros de l'innovation industrielle
}

\author{
Pierre Bézier and the Renault Transfer Machines (1940-1960). When \\ Cinema Fails to Glorify an Industrial Hero
}

\author{
Alain P. Michel ${ }^{1}$ \\ ${ }^{1}$ IDHE.S, Univ Evry / Université Paris Saclay, France, alain.michel@univ-evry.fr
}

RÉSUMÉ. Pierre Bézier est un anti-héros de l'innovation industrielle. II a été tour à tour l'artisan d'une forme originale d'automatisation, le promoteur en Europe de la commande numérique, puis l'un des fondateurs, à l'échelle mondiale, de l'informatique industrielle. Les "courbes et surfaces " auxquelles il a donné son nom sont aujourd'hui encore massivement utilisées sans qu'au patronyme ne soit associé un personnage et sans relier sa dernière innovation aux deux précédentes, pratiquement tombées dans l'oubli. Le papier propose d'analyser l'échec de la campagne de promotion cinématographique des machines transfert de Renault à la fin des années 1950. Ce cas permet de confronter les schémas de la pensée industrielle d'une époque aux pratiques réelles du terrain usinier. Autrement dit, lorsque la trajectoire de Bézier résiste aux cadres d'interprétations canoniques de l'héroïsme industrielle, l'anecdotique acquiert une valeur historique.

ABSTRACT. Pierre Bézier is an anti-hero of industrial innovation. He has alternately been seen as the artisan of an original form of automation, the promoter in Europe of numerical control, and as one of the founders of industrial computing. While "Bézier curves and surfaces" are still widely used, the family name is seldom associated with the man and hardly ever linked to his previous innovations which have fallen into oblivion. This paper investigates the failure of a film promotion campaign for Renault transfer machinery in the late 1950s. This case study makes it possible to compare Bézier's workshop practices to the patterns of industrial thought which did not consider him as a worthwhile innovator. In other words, as Bezier's trajectory resists the canonical interpretative frameworks of heroic industrial innovation, the anecdotal acquires a historical value.

MOTS-CLÉS. Automatisation, mécanisation, commande, électromécanique, numérique, film, publicité, ingénieur, entrepreneur, routine.

KEYWORDS. Automation, mechanization, control, electromechanical, digital, film, advertising, engineer, contractor, routine.

\section{Introduction}

Pierre Bézier est un anti-héros de l'innovation industrielle. Peu de gens le connaissent alors qu'il a été tour à tour l'artisan d'une forme originale d'automatisation (1947), le promoteur en Europe de la commande numérique (1958), puis l'un des fondateurs, à l'échelle mondiale, de l'informatique industrielle (1966). C'est cette dernière innovation qui lui est habituellement attribuée à l'exclusion des deux précédentes, pratiquement tombées dans l'oubli. Comme le bourgeois gentilhomme, nous manions du «Bézier » sans le savoir. Les «courbes et surfaces » auxquelles il a donné son nom sont aujourd'hui encore massivement utilisées. Elles sont au fondement de la Conception et fabrication assistées par ordinateur (CFAO) qui réorganise la production de série et redessine le monde virtuel. Elles sont un outil banal de nos pratiques informatiques quotidiennes sans que nous ayons besoin de connaitre l'origine de l'algorithme conçu cinquante ans plus tôt, dans un cadre collectif et pour un tout autre dessein.

Pourquoi Pierre Bézier est-il si mal connu et n'a-t-il laissé à la postérité que son nom propre ? Et pourquoi la première innovation de Pierre Bézier, celle des machines transfert à têtes 
électromécaniques a-t-elle été si rapidement oubliée ? Plutôt que de s'en plaindre ou de vouloir réparer une méconnaissance, je propose de considérer cet oubli comme un « cas » susceptible de questionner la nature de l'innovation industrielle du milieu du XXème siècle, époque où l'invention est habituellement collective $(\mathrm{R} \& \mathrm{D})$, initialement confidentielle et rapidement décrétée caduque. La préservation du secret assure un temps l'avantage concurrentiel de l'entreprise. Sa promotion dans l'urgence sert aussi bien la réputation de l'entreprise que le profit à tirer d'une modernité rapidement dépassée.

Dans cette perspective, les traces qui subsistent des activités inventives de Pierre Bézier sont autant d'indices permettant de résoudre l'énigme d'une renommée paradoxalement obscure. Ces sources matérielles et visuelles permettent d'entrer dans la «boîte noire» de la capacité d'innovation d'une époque afin d'y chercher les causes de phénomènes qui sortent des schémas d'explications habituelles. J'analyserai successivement une campagne de promotion cinématographique des années 1950, la cohérence du parcours d'un ingénieur-innovateur surprenant et enfin les spécificités antihéroïques d'une époque rétrospectivement dite « glorieuse».

\section{La célébration cinématographique d'une innovation invisible}

Les machines (à) transfert de la Régie Renault sont mises en place à partir d'avril 1947 dans le cadre de la «bataille de la 4 CV » [FRI 79]. Soutenus par le nouveau PdG de la Régie, Pierre Lefaucheux, les «modernisateurs » ont eu à faire face au scepticisme d'une part de la direction et palier les pénuries de cette période de reconstruction [MIC 07]. La promotion cinématographique de ces automatismes industriels a lieu sept ans plus tard alors que le dispositif innovant au lendemain de la guerre, est en train d'être dépassé par un nouveau système de commandes numériques [NOB 84]. Ce n'est qu'une fois la lutte des « chaînes transfert » [NIG 06].

En mars 1950, la Régie crée le «Service de cinéma industriel » [FAR 91][NIG 06]. L'entreprise croit d'abord aux capacités du cinéma à améliorer les performances industrielles ${ }^{1}$. A partir de 1953, la mission du Service cinéma change. La dimension ergonomique des petites séquences « d'études du travail humain » est mise de côté. La production des films de l'entreprise vise un plus large public. On observe une convergence d'intérêts pour une représentation cinématographique des machines transfert de Renault. En 1954, un film institutionnel du ministère de l'Education nationale a implicitement pour mission de les promouvoir [KLI 54]. En ouverture du documentaire, le procédé du «travail à la chaîne » est présenté comme une évidence de la vie quotidienne, puis son application est déclinée dans différentes industries : pharmacie, faïencerie, cordonnerie, confection, petite métallurgie, horlogerie et machines à écrire. Le cas de l'industrie automobile n'est pas présenté dans ce film, mais il suggère que le travail à la chaîne débouche naturellement sur l'utilisation des «machines-transferts » (sic) automatiques mises au point chez Renault.

\footnotetext{
${ }^{1}$ Jean Farcy est recruté par Jean Myon pour «mettre le cinéma au service de la formation ». Son premier «client» est le service méthodes organisation de Marcel Delfosse qui se sert du cinéma «pour développer les nouvelles méthodes de travail ». Voir Jean Farcy, «Renault et le cinéma... », art. cit.
} 


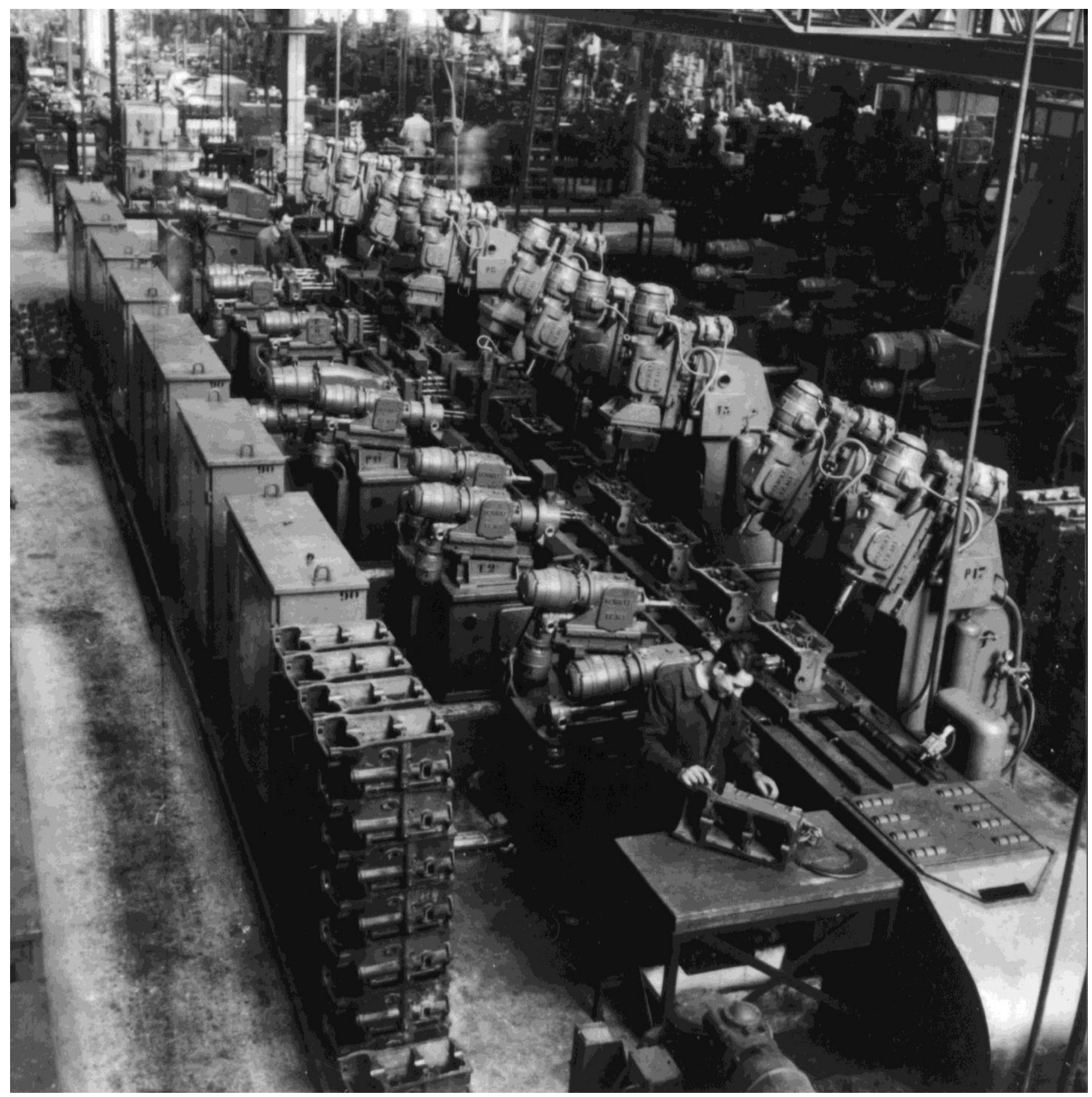

Figure 1. Vue plongeante de l'atelier U5 montrant la machine transfert d'usinage des carters-cylindres de la 4 CV (avril 1948) (c) Renault communication / RD / PHOTOGRAPHE INCONNU

La même année, un moyen métrage de fiction sert lui aussi à promouvoir la modernité de la Régie nationale [BLO 54]. Le film met en scène les péripéties de Pierrot, un enfant espiègle passionné de voiture, qui désobéissant à son père (joué par Louis de Funès) part à la recherche de son parrain, ouvrier fondeur à Billancourt. Ce périple clandestin d'un enfant dans l'usine, permet de redécouvrir les différents départements et ateliers de Billancourt et tout particulièrement les machines transfert. Caché dans un chariot, Pierrot croise un groupe qui visite l'atelier U5 et entend :

- Commentaire du guide : « et nous voici maintenant devant la plus grande de nos machines transfert. Machine automatique construite par nous pour l'usinage en grande série des pièces de moteur. » 
-Voix off : «Ils sont marrants les gosses. Rien ne les épate plus. On dirait que c'est eux qui ont inventé l'électricité »

- Commentaire du guide : «Et voici une tête électromécanique.»

-Voix off : « ... c'est vrai. Ils regardent la tour Eiffel et ils disent : «c'est vrai qu'avec 500 $m$ de plus, ça aurait peut-être eu l'air de quelque chose »...».

- Commentaire du guide : " ...et vous remarquez que tous les outils travaillent ensemble. Quand ils ont tous fini leur opération, les pièces avancent automatiquement à la station suivante. Et le cycle recommence. Vous comprenez ainsi le nom de ces machines. Les pièces y sont transférées toutes seules. Les têtes électromécaniques attaquent les pièces dans n'importe quelle position. Ces pièces ressortent à l'extrémité complètement usinée. Une machine transfert comme celle-ci peut accomplir jusqu'à 160 opérations en même temps. Les têtes électromécaniques agissent comme des robots. On croirait qu'elles voient, qu'elles entendent. En tout cas, elles savent ce qu'elles doivent faire. Et on ne peut les tromper, car elles ont de la mémoire ».

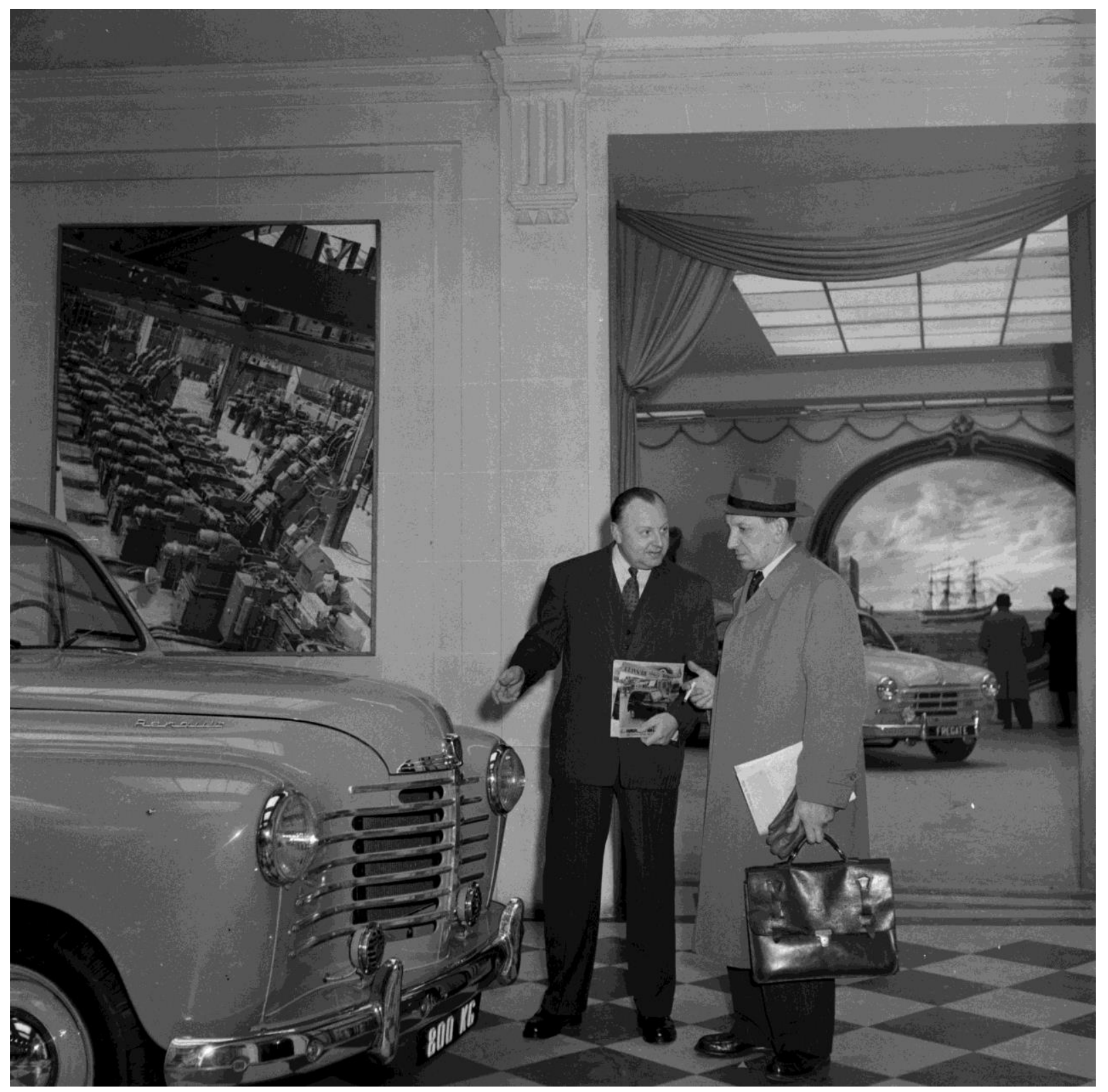

Figure 2. Affiche de promotion des machines transfert dans le Hall de vente du magasin des Champs-Elysées (1951) () Renault communication / RD / PHOTOGRAPHE INCONNU 
Ce film de fiction participe d'un retournement dans la conception de la publicité. L'aspect ludique de l'automobile est mis en valeur, avec la création de concours au cours desquels de futurs clients gagnent une 4 CV. C'est le moment où le Service publicité, dirigé par Paul Grémont depuis 1944, transforme la tonalité des réclames de Renault.

A la fin des années 1940, le message publicitaire met l'accent sur les arguments économiques et techniques d'une $4 \mathrm{CV}$ qui a encore peu de concurrents. L'engouement pour les concours visant à gagner une $4 \mathrm{CV}$ signale la valeur d'une voiture encore difficile à obtenir. Les acteurs de cinéma comme Jean Marais sont mis à contribution pour maintenir l'intérêt du public. En 1954, la cible visée est moins l'acquisition de l'objet que les avantages de son utilisation [GRE 85]'.

Au début des années 1950, la modernité des moyens de fabrication de Renault-Billancourt est un moyen de marquer la différence avec les modèles des autres constructeurs. Une photographie grand format d'une chaîne transfert est affichée dans le hall d'exposition des Champs-Elysées [LOU 10]. La Régie produit alors des documentaires industriels. En 1956, un film de promotion, intitulé simplement Machine transfert, donne une description technique des installations phares de la Régie [ANO 56]. Il établit une comparaison systématique des postes de travail entre «les anciennes chaînes » d'usinage des carters cylindre et la «nouvelle chaîne » transfert de la 4 CV. Dans les commentaires du film, le mot «chaîne » a une connotation positive, mais il change de sens selon l'âge du procédé auquel il est associé. Le commentaire distingue d'un côté la chaîne moderne que représente la machine transfert fonctionnant apparemment sans hommes et de l'autre, la voix off insinue que les chaînes fonctionnant avec des ouvriers sont dépassées même si l'entreprise les améliore. La tonalité du documentaire de 1956 rejoint les appréciations d'Alain Touraine en 1955.

«D'un coté, des machines hors d'âge, inégalement engagées, des ateliers encombrés de stocks, des chaînes où s'usinent, mêlées, des pièces de divers modèles. De l'autre, des ateliers consacrés à un seul modèle, la $4 \mathrm{CV}$,où dès que la cadence normale est atteinte, l'engagement des machines est presque parfait et où les stocks, sans être complètement éliminés, sont très réduits. Ce contraste traduit celui de l'ancienne et de la nouvelle organisation économique» [TOU 55].

Le cinéaste et le sociologue défendent le même point de vue optimiste que les outilleurs.

Trois ans plus tard un autre documentaire est intitulé simplement U5 du nom de l'atelier d'usinage d'origine des TM. Pour son réalisateur André Cantenys et pour le Service cinéma de la Régie, le dispositif est tellement connu que ces deux signes suffisent à désigner l'ensemble [CAN 59]. Le commentaire, toujours aussi docte, témoigne d'une hésitation sur le vocabulaire utilisé pour qualifier les installations : «chaîne », «ligne » et « convoyeur ». On observe également une insistance sur le contrôle et la responsabilité de l'équipe :

«A la sortie, un dispositif de poinçonnage inscrit le matricule de l'équipe qui porte la responsabilité ».

En 1959, l'automatisation complète ne va plus de soi. Les films traduisent un souci de réintroduire l'humain en gage de la qualité. L'évolution de leur message est également perceptible dans les nuances entre deux versions d'un même film de promotion de la Régie destiné à un public plus large que les documentaires techniques. En 1957, 24 heures à la Régie propose un panorama des activités de la Régie à Billancourt, dans les usines décentralisées et à travers le monde et laisse une large place aux

\footnotetext{
${ }^{2}$ En 1955, Paul Grémont effectue un voyage en Amérique pour s’inspirer des méthodes américaines.
} 
images d'usinages automatiques des machines transfert qui font la gloire de la Régie Renault [SIR 57]. Le commentaire toujours aussi calme et didactique énumère dans le détail les nombreux éléments qui participent au système technique. La fabrication automatique est ainsi magnifiée :

«Prodigieuse création des techniciens de la Régie (...), 625 outils attaquent, ensemble le métal alimenté en pièces par 850 mètres de convoyeurs à aiguillages et ascenseurs pneumatiques $»^{3}$.

A la fin de cette ligne transfert, les carters cylindre sortent usinés et le commentaire répète simplement : « 40 secondes : une pièce. 40 secondes : une pièce ». En 1961, une seconde version de ce même film réutilise les mêmes images, mais avec un commentaire qui suggère que la cadence a été augmentée : «30 secondes : une pièce. 30 secondes : une pièce ». La nuance échappe au grand public, mais l'augmentation est conséquente et indique l'un des moyens choisis entre 1957 et 1961 pour répondre à la hausse de la production voulue par la direction. Or, pour que les mêmes machines usinent plus vite, il faut avoir amélioré la qualité des outils coupants. Nous y reviendrons.

La principale nouveauté de ces films de la seconde partie des années 1950 réside dans cette perspective d'automatisation du travail, c'est-à-dire le fait de remplacer l'homme par la machine. «La machine transfert devient une vedette cinématographique, dont la caméra montre avec un grand luxe de détails les combinaisons de manutention automatique, d'usinage proprement dit et de contrôle, soit une succession impressionnante de mouvements de translation verticale et horizontale... » [NIG 06]. Fort de cette conception de la modernité, l'action des ingénieurs mécaniciens s'épanouit en combinant des impératifs d'efficacité avec leur plaisir de participer à des prouesses technologiques. Ils ont la possibilité de concevoir et de mettre au point des procédés de fabrication extraordinairement sophistiqués, spectaculaires, mais coûteux et souvent moins fiables et rentables que prévus.

Or en même temps que la chaîne transfert triomphe dans les films institutionnels et dans la thèse d'Alain Touraine un retournement d'appréciation s'opère à propos du travail à la chaîne dans d'autres films. En 1956, Jean Mitry réalise un film critique contre les méfaits de la mécanisation tout en prônant, par des commentaires emphatiques, les bienfaits de la science et de l'automatisation :

«Pris dans l'engrenage, l'être humain devient un maillon d'une chaîne, un rouage dans un ensemble mécanique ». Le film conclut : «après une telle journée, on n'a plus la force de sourire, de penser d'aimer » [MIT 56].

Par rapport au film de propagande gouvernementale de 1954, Le travail à la chaîne, le panorama historique de Mitry est nettement plus critique à propos du processus de mécanisation. Comme chez Touraine, l'automatisation est présentée comme un moyen de libérer les travailleurs des besognes fastidieuses. Le documentaire de 1956 pose explicitement le problème du rapport des hommes aux machines et ne présente pas les perspectives d'une société harmonieuses comme une évolution évidente. A la même époque, tous les films d'entreprise évitent d'aborder cette question du rôle des hommes travaillant sur les chaînes. Même pour les installations modernes, la chaîne semble commencer à déranger.

Les premières représentations cinématographiques des machines transfert Renault sont décalées par rapport à la chronologie de leur mise en place. Ce dispositif de pointe a été promu un temps sans être dévoilé, puis révélé au public et aux clients industriels potentiels au moment où il était devenu banal ce

\footnotetext{
${ }^{3}$ De même, dans U5 d'André Cantenys : «Le moteur qui vient de passer au banc d'essai quitte U5, un atelier considéré comme l'un des plus automatisé du monde. C'est à U5 que les groupes d'organes principaux ont été assemblés autour du bloc cylindre ».
} 
qui ne signifie pas pour autant qu'il ne continue pas à fonctionner efficacement dans les usines de Renault et ailleurs. Si la campagne de promotion cinématographique des machines transfert a été brusquement stoppée à la fin des années 1950, son objectif commercial immédiat a été atteint et les machines transfert à TEM sont restée au cœur des opérations d'usinages modernes, chez Renault et ailleurs. A l'échelle industriel, leur oubli est relatif. C'est plus par rapport à leur inventeur, et en lien avec le parcours professionnel de Bézier qu'il est pertinent de nous interroger.

\section{Le parcours déroutant d'un innovateur-salarié}

Ce qui a échoué, c'est la tentative de réhabilitation personnelle de Pierre Bézier. Quand il prend sa retraite en 1975, il tente dans la publication des «souvenirs d'un outilleur » de raviver la mémoire de sa première innovation. Il l'avait déjà fait, de manière plus confidentielle, à l'occasion de sa candidature à la chaire de Fabrication mécanique du CNAM en mai 1968 en rédigeant une notice typographiée de quinze pages. Ce «curriculum vitae complet» met en valeur une expérience professionnelle hors du commun et en propose une synthèse :

« Au cours de 35 ans de carrière, j'ai été appelé à prendre part à de nombreux travaux ou à les diriger. Leur énumération serait fastidieuse et peu significative et je crois plus utile de préciser les tendances principales de ma conception des fabrications, et de décrire les options dont, à plusieurs reprises, j'ai pris la responsabilité » [BEZ 68].

Il classe ses travaux en six domaines correspondant à autant de types d'amélioration de pratiques ou de process. Ce panorama vise à souligner à la fois la continuité et la complémentarité de ses domaines d'expertise.

La première époque (1927-1934) est celle des dispositifs cinématiques pour lesquels il a été formé à l'Ecole des Arts et Métiers (Paris, 1927) comme l'ont été son père et son grand-père. Ce gadzarts est issu d'une dynastie d'origine ouvrière, catholique et conservatrice, dont l'ascension sociale suit l'affermissement de la IIIè République. Rentré major et sorti second de sa promotion, sa « Médaille d'argent » lui permet de compléter sa formation d'outilleur à l'Ecole supérieure d'électricité qu'il intègre sur examen probatoire en septembre 1930 pour en sortir au «cinquième rang ». Il cumule donc un «Brevet» d'ingénieur mécanicien (22 juillet 1930) et un «Diplôme » d'ingénieur électricien (juillet 1931) consolidant ainsi non seulement ses compétences techniques, mais également sa légitimité professionnelle. Pierre Bézier fait ensuite un service militaire d'un an (1931-1932), passant par l'École d'artillerie de Poitiers où il se spécialise dans la topographie ${ }^{4}$. Sa triple formation de mécanicien, («gadzarts»), d'électricien («supélec») et d'artilleur («diable noir ») s'avèrera déterminante pour la suite. Il entre chez Renault à l'atelier d'outillage central (AOC) peu de temps après que Louis Renault a inauguré sa nouvelle usine sur l'Ile Seguin. Spécialiste de la fabrication mécanique et des outillages, il devient rapidement dessinateur projeteur au bureau d'études d'outillages mécaniques (BEOM) sous la direction d'Etienne Coindeau, un gadzarts comme lui.

La seconde époque (1934-1939) est celle des premières installations de commandes «électromécaniques ». Bézier est alors chef d'une section du BEOM, c'est-à-dire qu'à la tête d'une équipe de 5 ou 6 dessinateurs, il est chargé de mener quelques projets visant à transformer la nature de dispositifs automatiques traditionnels. Dès lors, ses inventions s'inscrivent dans des collectifs, ceux des équipes qu'il est emmené à diriger au sein d'un ensemble plus vaste qu'est la firme. Alors que la crise économique mondiale commence à faire sentir ses effets à Billancourt, ses missions consistent surtout

${ }^{4}$ «Date d'incorporation: 15 octobre $1931 »:$ Demande de dossier militaire, Chemise « Personnel », Dossier Bézier, Archives du CNAM. 
à améliorer et à adapter les machines-outils existantes qui sont pour l'immense majorité à commandes cinématiques et à puissance hydraulique : il saisit cette occasion pour concevoir de nouvelles formes économes d'automatismes électromécaniques. Ces substitutions s'apparentent au combat d'un électromécanicien spécialiste d'améliorations frugales.

La guerre et la libération marque une troisième période (1939-1947). L'arrêt de l'activité automobile ordinaire offre à Bézier le temps nécessaire pour imaginer et étudier la systématisation des dispositifs d'automatismes expérimentés de manière ponctuelle avant-guerre [GRE 17]. Bézier devance l'appel en août 1939 pour rejoindre son régiment d'Artillerie. Prisonnier à la suite de la débâcle de mai-juin 1940, c'est en captivité qu'il conçoit le principe de la tête électromécanique (TEM) à «commandes séquentielles » destinée à être l'unité de base d'un mécanisme inédit de production automatique. Libéré en mars 1941 dans le cadre de politique dite de la Relève, il rejoint le bureau des méthodes de Renault où il poursuit confidentiellement, dans une usine occupée et plusieurs fois bombardée, l'étude de la TEM et la fabrication des premiers prototypes. A la Libération, au sein de la nouvelle Régie nationale des usines Renault (RNUR), il devient chef du BEOM : il est alors en bonne position pour défendre cette première innovation qui sera portée à son crédit. C'est ce qu'il appelle dans sa Notice de 1968, les «machines [sous-entendu électromécaniques] à éléments normalisés ».

La période 1947-1952 est celle de l'application à la grande série des «machines (à) transfert » équipées de TEM qui étonneront même les Américains. Ces TEM sont conçues pour être «standards et flexibles », c'est-à-dire interchangeables et réutilisables sur différents types de dispositifs. Dans le cadre de la «bataille pour la $4 \mathrm{CV}$ » le dispositif expérimental devient un instrument de la fabrication de masse. Sa mise en place puis sa montée en cadence sont laborieuses, mais elles permettent relativement rapidement la fabrication du nouveau modèle populaire de voiture Renault et assurent le succès de la Régie.

Durant l'époque (1952-1960) du triomphe (apparent) de Pierre Bézier, sous la présidence de Pierre Lefaucheux jusqu'en 1955, le directeur des Etudes mécaniques tente de généraliser l'usage des machines transfert - au-delà de l'usinage -, vers les opérations de stockage puis d'assemblage :

«Alors que les moyens automatiques d'usinage avaient fait de grands progrès, force était de constater que, vers 1960, les moyens d'assemblage restaient, à quelques détails près, semblables à ceux de $1920 »[$ BEZ 68].

C'est aussi le moment où les commandes numériques sont exposées dans les expositions puis dans les usines américaines [NOB 84]. Au sein d'une nouvelle Division Machine-outil (DMO), dont la direction lui est confiée, Bézier propose de substituer les $\mathrm{CN}$ aux commandes électromécaniques :

«Persuadé, dès le premier abord, de l'importance que la commande numérique était appelée à jouer (...), j'ai dirigé l'étude... »[BEZ 68].

C'est au cours de la dernière partie de sa carrière (1960-1975) qu'il présente sa candidature pour la Chaire du CNAM. Sa notice de mai 1968 ne fait pas explicitement état du système « Unisurf », logiciel de conception et fabrication assisté par ordinateur (CFAO) dont le développement est alors déjà bien engagé dans la confidentialité des études de carrosserie. Le brevet a été déposé en 1966 et depuis avril 1968, les prototypes de planche à dessin et de fraiseuse commencent à fonctionner au centre de recherche de la Régie à Rueil :

« La solution que j'ai proposée en 1965 a paru suffisamment intéressante à la DGRST pour que celle-ci, en 1966, nous accorde son soutien. Deux ans plus tard, une machine à dessiner et une fraiseuse tri-dimensionnelle fonctionnaient. Cette réalisation a demandé la coordination de travaux relevant de plusieurs domaines : mathématiques, calcul automatique, électronique, 
asservissements, carrosserie, emboutissage, tracé au grand plan, usinage des outillages de tôlerie, etc. Il est possible que cette méthode ait des conséquences dans l'emploi de l'électroérosion ou de l'électrolyse pour la fabrication des matrices de forges, des coquilles de fonderie et des outils d'emboutissage » [BEZ 68].

En janvier 1968 Pierre Bézier a créé la sensation en exposant les principes d'Unisurf lors du congrès de la Society of Automotive Engineers (SAE) à Detroit [BEZ 68-b]. Dans sa Notice, il parle de calcul « automatique » mais pas d'ordinateur. Il utilise encore le terme de «numérique », mais il sait déjà que c'est par rapport au processus de Computer aided design (CAD) que son exposé de Detroit fait des vagues. Le curriculum vitae reste donc allusif concernant les recherches entreprises chez Renault ces huit dernières années. Le candidat ne dit pas non plus que depuis octobre 1960, il a été déchargé de ses responsabilités dans le secteur de la machine-outil pour être nommé Directeur attaché à la Direction générale des fabrications, titre qui suggère qu'il est placé au sommet de la Fabrication mais aussi qu'il a été extrait de son milieu de mécanicien en charge de la prospective technique dans le monde des carrossiers. Paradoxalement, ce changement de responsabilité, qu'il vit comme une mise à l'écart, lui donne une seconde occasion de se consacrer à une réflexion de fond et de mener à bien, jusqu'à sa retraite, la mise au point puis le développement de la CFAO [POI 88].

Ce contexte de mise «au placard» lui donne également la disponibilité pour candidater à la nouvelle chaire de Fabrications mécaniques. Dans son texte de candidature Bézier fait de plus en plus usage de la première personne du singulier à mesure qu'il décrit ses actions récentes. La récurrence du «je » suggère la position bancale d'un directeur marginalisé dans son entreprise, mais dont la réputation internationale est devenue incontestable lorsqu'il postule. L'ingénieur-candidat-professeur prend de la hauteur vis-à-vis d'une mutation technique en cours et à laquelle il a participé depuis 35 ans. Il met l'accent sur les continuités plutôt que sur les ruptures en s'affranchissant des repères habituels de l'histoire des automatismes industriels. Mais pour candidater à une chaire de fabrications mécaniques, mieux vaut ne pas brouiller les pistes (ou inquiéter les enseignants en fonction). Ses propos sont biaisés par les objectifs du candidat et par son souci de gommer la déchéance professionnelle d'octobre 1960 grâce à son triomphe américain de l'hiver 1968.

Bézier place sa première innovation, celle des TEM, comme l'aboutissement d'une succession de trois temps depuis les machines-outils cinématiques de base, les premières expériences automatismes électromécaniques jusqu'aux automatisations de grande série de la fin des années 1940. Il insinue aussi que sa seconde « idée bizarre », celle de la CFAO, se place dans la lignée des actions précédentes, celle de la «manutention et stockage automatiques » qu'il date de 1956, puis «l'assemblage automatique » vers 1958 et la «commande numérique » avant 1960. Mais, là où lui défend la cohérence d'un parcours créatif, ses contemporains perçoivent une série de ruptures inventives. Ces tentatives de justification sont, au mieux, perçues comme une opération pathétique de réhabilitation, au pire comme la confirmation de l'incohérence d'un innovateur en porte-à-faux vis-à-vis d'un monde qui se modernise.

\section{L'impossible reconnaissance d'un génie créatif hors normes}

Entré le 1er mars 1933 à la Société Anonyme des Usines Renault où il achève sa carrière en 1975, Pierre Bézier n'œuvre pas dans une période propice à en faire un héros industriel. Comme membre d'une usine, il s'inscrit d'abord dans un collectif où ni les relations professionnelles ni les rapports aux techniques ne sont «limpides». Comme techniciens de l'industrialisation, Bézier et ses collègues n'ont pas besoin que d'autres leur disent leur valeur pour la reconnaitre. La fonction hérö̈que change d'arène[GAR 16].

Bézier nait au moment où les sociologues ont posé un triple interdit sur le «fantasme de la chronologie », sur le primat du tout politique et sur «l'idole biographique » [SIM 03]. L'école des 
Annales confirme, durant les années 1920, ce rejet de l'individuel au profit du collectif, l'interdit contribuant à faire de l'approche biographique un tabou et de la biographie un «mot-totem ». Dans ces mêmes années, Joseph Schumpeter tente de réhabiliter la figure canonique de l'entrepreneur-innovant à laquelle l'ingénieur salarié peut difficilement prétendre. L'économiste autrichien attribue à l'entrepreneur-innovant un rôle moteur dans le développement de l'économie capitaliste [SCH 11] [SCH 28] [GIS 12]. L'entrepreneur est celui qui « administre l'œuvre de production » et pas forcément le propriétaire des capitaux. La fonction d'entrepreneur est distincte de celles du patron-capitaliste et du distributeur de travail avec lesquelles elle peut se combiner. A la différence du patron-employeur voire du sous-traitant, l'action économique de l'entrepreneur-innovateur porte d'abord sur l'invention de produits ou de procédés et secondairement sur la hausse de la production ou du capital : elle n'est pas tant quantitative que qualitative. Par ses innovations, l'entrepreneur schumpétérien brise les routines du monde statique des gestionnaires et des bureaucrates. C'est en cela que Schumpeter lui attribue un rôle héroïque de capitaine d'industrie moderne («moderner Industriekapitän ») [SCH 28-b] [LEB 14]. Ce lien entre la notion d'innovation et la figure de l'entrepreneur reste ancré dans l'histoire technique de l'innovation et plus encore dans l'histoire économique des affaires [CEC 07]. Cette mise en valeur de l'entrepreneur s'inscrit donc dans une réflexion profonde autour de la notion de commandement « devenu un problème » voire une «obsession » selon Yves Cohen $[\mathrm{COH} 13]^{5}$.

Depuis les années 1920, l'hérö̈sme entrepreneurial est en crise face aux doutes de la «modernité désenchantée » qui parcours l'occident industriel [FUR 15]. Le scepticisme imprègne en profondeur la société en même temps qu'il tend à s'y diluer. Dans son roman satirique Babbitt de 1922, Sinclair Lewis transpose le modèle du héros américain dans la banalité d'un groupe de "purs » hommes d'affaires (salesmen) du Middle-west [LEW 22] [HUN 10]. L'auteur reçoit le prix Nobel en 1930. A cette occasion le poète suédois Erik Axel Karlfedt célèbre en Georges F. Babbitt la personnification du «héros populaire américain de la classe moyenne $»^{6}$. Les déboires et désillusions du personnage souligne la fragilité du self made man produit par le rêve américain. En Amérique, la satire résonne tout particulièrement. Les milieux d'affaires doivent répondre aux arguments d'une seconde génération de polémistes, les muckrackers qui, face à l'essor des compagnies géantes et s'appuyant sur les lois anti-trust, défendent l'idée que seul le « petit » est bon et que l'action politique doit juguler l'expansion des grandes entreprises considérées comme mauvaises car faussant les mécanismes de la concurrence par les Ententes et les Cartels [RII 90] [JOS 34]. L'analyse de Schumpeter sur la nature du capitalisme moderne intervient opportunément dans un climat culturel hostile aux grandes entreprises. Ce n'est donc pas par hasard que sa théorie libérale de l'action entrepreneuriale est accueillie au Centre de recherche en histoire des entreprises de l'université Harvard créé en 1927. Schumpeter y enseigne, ponctuellement dans un premier temps (1927-1928 et 1930), puis régulièrement après son installation aux Etats-Unis en 1932. La notion d'innovation et l'action d'acteurs nouveaux dans la dynamique économique viennent à l'appui de l'opération de réhabilitation engagée à Harvard. Mais ce personnage, archétype de l'entrepreneur-innovant, est déjà un mythe qui se nourrit de quelques « héros » du temps des pionniers et des premiers entrants (prime-movers). Bézier débute lui sa carrière dans le monde de l'automobile en période de crise et bien après le temps des précurseurs. Il ne fait pas partie du milieu des ingénieurs-innovateurs schumpétériens de la génération précédente qui sont choisis pour être le sujet d'une biographie. Bézier n'en partage pas moins un esprit d'entrepreneur, dans sa façon de

\footnotetext{
${ }^{5}$ L'obsession du chef (pas seulement celle du « grand chef ») n'était pas propre aux totalitarismes et à l'affirmation de la gouvernementalité. Yves Cohen distingue une histoire de pratiques discursives qui dessinent les contours des conceptions variées de l'autorité et une histoire de pratiques opérationnelles qui recourent à des instruments spécifiques (plans, visite, écrits d'action, etc.).
}

${ }^{6}$ Erik Axel Karlfedt, «Presentation Speech », December 10, 1930. A l'occasion de la remise du Prix Nobel de littérature. 
«prendre » en charge ce qu'il considère comme une mission d'innovation. L'horizon de son combat n'est pas celui d'une entreprise globalement viable.

Jusqu'en 1944, les activités innovantes du jeune ingénieur sont discrètes: en période de crise économique puis de guerre et d'occupation elles s'apparentent plus aux améliorations ordinaires des bureaux d'études mais elles préparent les innovations spectaculaires de l'après-guerre. Celles-ci se déploient pendant la période que Jean Fourastié a qualifié en 1979 de «Trente Glorieuses », au cours desquelles «l'Etat entrepreneur » attribue au constructeur nationalisé un rôle de levier économique et d'usine-pilote des avancées sociales. Billancourt acquiert une réputation de «forteresse ouvrière » au moment où l'étendard du syndicalisme CGT se fissure. La Régie délocalise déjà une partie de ses activités productives polluantes, ce qui redistribue la part des « cols bleus » et des « cols blancs » dans la main-d'œuvre de Billancourt.

L'usine automobile est un système technique sophistiqué fait d'usinages et de montages, d'étaux limeurs et de rectifieuses, d'automatismes et de commandes au fonctionnement mystérieux dans des ateliers saturés et bruyants, selon des procédés mis en place dans la discrétion. Or, Bézier travaille dans les bureaux des Méthodes qui s'occupent du fonctionnement efficace de ce système technique ; plus que des découvertes spectaculaires, il cherche des solutions concrètes. De fait, il est polymorphe. Chef d'équipe, il est membre d'un collectif et non pas l'auteur individuel des projets qu'il dirige. Employé loyal, il œuvre pour le succès de son entreprise. Mécanicien scrupuleux, il défend avec fierté sa corporation. Patriote, il combat pour l'honneur de sa nation. La variété de ses compétences industrielles lui donne le sentiment d'intervenir à tous les échelons de l'usine. Il en fait une question de principe, fondée sur son interprétation de la sagesse populaire :

«D'après un proverbe arabe, on ne peut faire boire un chameau qui n'a pas soif; un individu ancré dans sa routine trouve toujours d'excellentes raisons de ne rien changer à sa façon de faire ou de ne rien faire. Pour surmonter l'obstacle (...), il n'y a qu'une solution : exécuter d'abord soi-même le travail (...). Mais il faut, pour employer la méthode, être capable, selon l'expression consacrée, de se retrousser les manches et d'attraper les manivelles » [BEZ 82].

L'usine est également traversée de tensions sociales qui s'expriment lors des grèves (avril 1947, mai 1968 , etc.) et se traduisent par d'autres pratiques plus discrètes de flânerie, freinage, retards ou absences. Elle est sujette à des incidents tels que les pannes, les loupés ou les accidents. Elle est le théâtre d'enjeux de pouvoirs et de rapports de forces mouvants entre les impératifs de rendement, des projets individuels, des alliances circonstancielles et des réseaux provisoirement stabilisés. L'ingénieur-innovateur contribue à l'excellence technologique française dans un cadre techniquement, socialement et politiquement tendu. Il reste en place au prix de luttes récurrentes autour d'inventions marquantes, telle la machine transfert. Dans ce milieu instable, l'innovation n'est pas toujours bien accueillie, ni par les organisations ouvrières inquiètes des effets de l'automatisation sur la maind'œuvre, ni par ceux que Bézier appelle «les gens sages», les «traditionnalistes » et autres «gens sérieux » [BEZ 75][BEZ 85]. Personnage pittoresque au caractère trempé et aux idées arrêtées, ses témoignages offrent un écho précieux de l'évolution technologique d'une période. Une personne, si singulière soit-elle, et dans la mesure où elle le reste, n'est jamais seule ni dans une position statique. L'expérience professionnelle de Pierre Bézier nous permet d'observer de l'intérieur quelques détails de cette société. Depuis ses liens avec la direction jusqu'aux rapports avec les ateliers d'usinages, il participe à la vie des bureaux d'études.

Mais comme tout récit de carrière rétrospectif, les réminiscences et témoignages qu'en donne Bézier sont partiels et partiaux. L'histoire a besoin de confronter les points de vue afin d'éclairer les zones d'ombres plutôt que les effacer, de documenter le cheminement sinueux et tumultueux des inventions plutôt qu'aplanir le récit de l'innovation. Cette chronique du parcours professionnel à rebondissements 
de Pierre Bézier mérite l'attention car elle donne l'occasion d'entrer dans la «boîte noire du phénomène technique »[ROS 82].

\section{Conclusion}

Pierre Bézier est un « antihéros » non pas à cause de son déficit de gloire, mais parce qu'il bouscule les critères de l'ingénieur-innovateur de son temps. L'inventeur donne son nom propre à l'algorithme d'une courbe «dont l'élégance dissimule la complexité » [BEZ 82]. Dans ses textes de réminiscences, le polémiste pointe des lieux de tensions, révèle des enjeux cachés, dénonce des incohérences et met en lumière «toutes les lézardes plus ou moins visibles » d'un monde qui l'entoure [HAR 01]. De son œuvre mécanique subsistent quelques machines rouillées, débris de mécanismes aujourd'hui muets [SER 94]. Les fragments de la chaîne transfert conservés à l'usine Renault de Cléon sont en attente d'une opération muséologique qui expliquera leur fonctionnement, maintenant que l'environnement technique qui les faisait fonctionner a disparu. Il reste pour cela quelques témoins ainsi que de multiples images en noir et blanc. Les clichés et les films de promotion rappellent la gloire d'une forme d'automatisation oubliée alors que d'autres machines transfert continuent d'usiner à Cléon.

Les fragments subsistants ne sont pas tous fortuits. Bézier conserve dans ses archives personnelles les documents qui appuient son analyse. Avec ses collègues techniciens, il investit dans les années 1970 la Section d'histoire des Usines Renault pour donner sa version du passé 7 . Dès sa retraite, il profite de chaque moment de triomphe personnel pour envoyer des piques à ceux qui ne l'on pas soutenu. L'ironie pourfend la langue de bois, l'allusion convoque des savoirs confidentiels, sans trahir les secrets industriels [BEZ 91] [BEZ 91-b]. A mots couverts, Bézier désigne des coupables et distribue les bons points sans qu'on puisse lui reprocher de fournir aux organisations ouvrières des arguments pour contester l'autorité de la direction. Le jeu est subtil. Une part des sous-entendus disparaît avec la mémoire des gens auxquels ils sont adressés [GIN 97]. Ainsi, Bézier affiche un signal à fouiller et décrypter.

Sa position en porte-à-faux vis-à-vis de son milieu crée des documents-monuments [LEG][RIC 91 97]. Leur recueil assemble les vestiges d'une vision idéologique du monde industriel. La mobilisation de sources plus variées révèle un homme aux multiples facettes, à la fois personnage pluriel et individu amer, roc inébranlable et château de sable mouvant. D'un côté il personnifie un monde industriel disparu et de l'autre, il témoigne en creux d'une singularité irréductible au collectif dont il est issu.

Par cette spécificité, le cas de Bézier permet de confronter les schémas de la pensée industrielle et des processus d'invention d'une époque (taylorisme, fordisme, retards, hybridation, etc.) aux pratiques réelles du terrain usinier. Autrement dit, lorsque la trajectoire de Bézier résiste aux cadres d'interprétations canoniques de l'organisation industrielle, l'anecdotique acquiert une valeur historique. Baignée dans les routines du milieu industriel, son œuvre peut être oubliée sans pour autant disparaitre. Dans ce contexte, il n'est pas étonnant que ses innovations soient évoquées en référence à des automatisme mystérieux, à une courbe sans visage, à une sommité sans reconnaissance académique, à un innovateur sans antécédences, pour déboucher sur une postérité technique anonyme.

${ }^{7}$ La Section d'histoire des Usines Renault est constituée en 1970 par Gilbert Hatry au sein du Club des agents de maîtrise, techniciens, employés des usines Renault (CAMTEUR). Elle publie en décembre 1970 le premier numéro de la revue De Renault Frères constructeurs d'automobiles à Renault Régie Nationale. La SHUR devient ensuite la Société d'histoire du groupe Renault (SHGR), puis Renault Histoire. 


\section{Références}

[ANO 56] Anonyme, Machines transfert, Service cinéma industriel, 1956.

[BEZ 68] Bézier Pierre, Notice sur les titres et travaux de M. Pierre Bézier, sans date (mai 1968), typographié, Archives de la Fondation des A\&M (Liancourt), Fonds Pierre Bézier, carton n ${ }^{\circ} 2$.

[BEZ 68-b] Bézier Pierre, «How Renault Uses Numerical Control for Car Body Design and Tooling », Automotive Engineering Congress, Detroit, Michigan, January 8-12, 1968, 7 p.

[BEZ 75] Bézier Pierre, «Souvenirs d'un outilleur », De Renault Frères, constructeurs d'automobiles, à Renault Régie Nationale, $\mathrm{n}^{\circ} 11$, décembre 1975, p. 177 et 181.

[BEZ 82] Bézier Pierre, «Petite histoire d'une idée bizarre (1)», De Renault Frères, constructeurs d'automobiles, à Renault Régie Nationale, $\mathrm{n}^{\circ}$ 24, juin 1982, p. 257.

[BEZ 85] Bézier Pierre, "Souvenirs d'un outilleur: de la 4 CV à la Dauphine », De Renault Frères, constructeurs d'automobiles, à Renault Régie Nationale, n 30, juin 1985, p. 144.

[BEZ 91] Bézier Pierre, « Humour : accommodements », Arts et Métiers, $n^{\circ}$ 156, 1991, p. 67.

[BEZ 91-b] Bézier Pierre, « Humour : accommodements », Arts et Métiers, $n^{\circ}$ 158, 1991, p. 53.

[BLO 54] Blondy Pierre, Champion Juniors, Franco-London production, 1954, 22 mn.

[CAN 59] Cantenys André, U5, Service cinéma de la Régie, 1959, 22 mn.

[CEC 07] Ceccarelli Marco (ed.), Distinguished Figures in Mechanism and Machine Science. Their contributions and Legacies, Part 1, Netherlands, Springer, 2007, 392 p. / Part 2, Springer, 2010, 259 p.

[COH 13] Cohen Yves, Le siècle des chefs. Une histoire transnationale du commandement et de l'autorité (1890-1940), Paris, éd. Amsterdam, 2013, p. 12.

[FAR 91] Farcy Jean, « Renault et le cinéma d'entreprise », Renault Histoire, n 3, juin 1991, p. 21-68.

[FRI 79] Fridenson Patrick, «La bataille de la 4 CV Renault », De Renault Frères constructeurs d'automobiles à Renault Régie Nationale, tome 3, $\mathrm{n}^{\circ}$ 18, juin 1979.

[FUR 15 Fureix] Emmanuel, Jarrige François, La modernité désenchantée. Relire l'histoire du XIXe siècle français, Paris, La découverte, 2015.

[GAR 16] Garçon Anne-Françoise, «La technique héroïsante : histoire d'un déni anthropologique et de ses risques », Les cahiers psychologie politique [En ligne], numéro 29, Juillet 2016. URL : http://lodel.irevues.inist.fr/cahierspsychologiepolitique/index.php?id=3319.

[GIN 97] Ginzburg Carlo, Le juge et l'historien. Considérations en marge du procès Sofri, Lagrasse, Editions Verdier, 1997 (1991).

[GIS 12] Gislain Jean-Jacques, «Les origines de l'entrepreneur schumpétérien », Revue Interventions économiques, $\mathrm{n}^{\circ}$ 46,2012

[GRE 85] Grémont Paul, «A bâtons rompus », De Renault Frères constructeurs d'automobiles à Renault Régie nationale, $\mathrm{n}^{\circ} 18-30$, juin 1979- juin 1985 .

[GRE 17] Grenard Fabrice, Le Bot, Florent, Perrin Cédric, Histoire économique de Vichy. L'Etat, les hommes, les entreprises, Paris, Perrin, 2017.

[HAR 01] Hartog François, Les XIXème siècle et l'Histoire. Le cas Fustel de Coulanges, Paris, PUF, 2001 (1988).

[HUN 10] Hunter Gordon, « Introduction », Sinclair Lewis, Babbitt, New York, Oxford University Press, 2010.

[JOS 34] Josephson Matthew, The Robber Barons: The Great American Capitalists, 1861-1901, New York, Harcourt, Brace and Company, 1934.

[KLI 54] Klimowicz W., Le travail à la chaîne, Ministère de l'Education nationale, Airfilm, 1954, 20 mn.

[LEB 14] Le Bot Florent, « Capitaines d'industrie », in Pierre-Marie Chauvin, Michel Grossetti, Pierre-Paul Zalio (dir.), Dictionnaire sociologique de l'entrepreneuriat, Paris, Presses de Sciences Po, 2014, p. 85-100.

[LEG] Le Goff Jacques, « Documento/nomumento », Enciclopedia Einaudi, Turin, G. Einaudi, vol. V, p. 38-48.

[LEW 22] Lewis Sinclair, Babbitt, San Diego, Harcourt, Brace \& Co, 1922. 
[LOU 10] Loubet Jean-Louis, Etienne Bernard, Michel Alain P., Les Champs-Elysées de Renault : 100 ans de présence sur la plus belle avenue du monde, Boulogne-Billancourt, Editions ETAI, 2010, p. 67.

[MIC 07] Michel Alain P., Travail à la chaîne : Renault 1898-1947, Boulogne-Billancourt, Editions ETAI, 2007.

[NIG 06] Nigwal (Nicolas Hatzfeld, Gwenaële Rot, Alain P. Michel), « Filmer le travail au nom de l'entreprise. Les films Renault sur les chaînes de production (1950-2005) », Entreprises et histoire, n 44, septembre 2006, p. $25-42$.

[NOB 84] Noble David, Forces of Production : A Social History of Industrial Automation, New York, Pantheon, 1984.

[MIT 56] Mitry Jean, La machine et l'homme, 1956, Films Armoria \& Cité Vox, 22 mn.

[POI 88] Poitou Jean-Pierre, Le cerveau de l'usine. Histoire des bureaux d'études Renault des origines à 1980. Recherche sur les conditions de l'innovation technique, Aix, Université de Provence, Publications Diffusion, 1988, p. 203.

[RIC 91] Ricoeur Paul, Temps et récit, Paris, Seuil, 1991.

[RII 90] Riis Jacob, How the other half lives. Studies among the Tenements of New York, New York, Charles Scribner's Sons, 1890.

[ROS 82] Rosenberg Nathan, Inside the Black Box : Technology and Economics, Cambridge, Cambridge University Press, 1982, p. vii.

[SCH 11] Schumpeter Joseph A., Théorie de l'évolution économique, (1911) 1926.

[SCH 28] Schumpeter Joseph A., Theorie der wirtschaftlichen Entwicklung (Théorie de l'évolution économique), 1911 \& 1926 J. A. Schumpeter, «The Instability of Capitalism », The Economic Journal, n 39, septembre 1928, p. 383.

[SCH 28-b] Schumpeter Joseph A., «Unternehmer», Handwörterbuch der Staatswissenschaften (4th ed.), Jena, G. Fischer, 1928, VIII, p. 476-487.

[SER 94] Séris Jean-Pierre, La technique, Paris, PUF, 1994.

[SIM 03] Simiand François, « Méthode historique et science sociale ( $2^{\text {ème }}$ partie) », Revue de synthèse historique, 1903, p. 129-157.

[SIR 57] Sirkis Jean-Jacques, 24 heures à la Régie, Service cinéma de la Régie, 1957, 22 mn.

[TOU 55] Touraine Alain, L'évolution du travail ouvrier aux usines Renault, Paris, Centre National de la Recherche Scientifique, 1955, p. 11. 